\title{
Gaze behavior in analytical and holistic face processing
}

\author{
GUDRUN SCHWARZER, SUSANNE HUBER, and THOMAS DÜMMLER \\ Friedrich Miescher Laboratory of the Max Planck Society, Tübingen, Germany
}

\begin{abstract}
We conducted two experiments examining children's and adults' gaze behavior when processing faces analytically (focusing on a single feature) or holistically (comparing the overall similarity of the faces). Children 6-8 and 9-10 years of age and adults were instructed to assign schematically drawn faces in Experiment 1 and photos of real faces in Experiment 2 to two categories. The categories were constructed so as to allow either an analytical or holistic categorization of the faces. During all trials, gaze behavior was recorded from stimulus onset until reaction. The location and duration of the fixations used were analyzed. Whereas the holistic processors fixated the whole area of the eyes and nose most and longest independently of age, analytical processors showed a more feature-specific gaze behavior, focusing their fixations upon the particular feature used for subsequent processing. Thus, differences in analytical and holistic face processing can be detected early in gaze behavior - that is, at the visual encoding stage.
\end{abstract}

Humans are experts at recognizing faces. We identify faces quickly and exactly without any effort. Small wonder, then, that extensive research has been carried out to understand the stages of information processing that underlie face-recognition performance. The two relevant stages of information processing are perceptual encoding and information processing. In the area of face processing, most studies have focused on the latter and have shown that people often use the configural processing mode-processing the relations among the facial features (e.g., Diamond \& Carey, 1986; Farah, Tanaka, \& Drain, 1995; Farah, Wilson, Drain, \& Tanaka, 1998; Freire, Lee, \& Symons, 2000; Tanaka \& Farah, 1993). Analytical face processing has been emphasized as being relevant, too (e.g., Bruyer \& Coget, 1987; Macho \& Leder, 1998; Schwarzer \& Massaro, 2001), and most recent studies concern the question of whether and how both configural and analytical information processing play a role in face recognition (e.g., Collishaw \& Hole, 2000; Leder \& Bruce, 2000; Schwaninger, Lobmaier, \& Collishaw, 2002; Searcy \& Bartlett, 1996; Tanaka \& Sengco, 1997). However, very few studies address the visual encoding stage in face processing directly and analyze what kind of facial information is extracted during face processing. According to Viviani (1990), the analysis of gaze behavior can be understood as a method to study the stage of visual encoding. Therefore, to understand the visual encoding stage of face processing, the present study investigated participants' gaze behavior when they processed faces analytically or configurally.

The authors are grateful to Nikolaus Troje for constructing and providing the facial stimuli in Experiment 2. Correspondence should be addressed to G. Schwarzer, University of Giessen, Otto-Behaghel-Str. 10F, 35394 Giessen, Germany (e-mail: gudrun.schwarzer@psychol. uni-giessen.de).

\section{Research on the Different Modes of Face Processing}

As mentioned above, the information processing modes people use to recognize faces is of both configural and analytical character. According to Maurer, Le Grand, and Mondloch (2002), configural processing can be divided into three types: (1) processing of first-order relationsthat is, seeing that a stimulus is a face because the features are arranged with two eyes above a nose, which is above a mouth; (2) holistic processing - that is, gluing together the features into a gestalt; and (3) processing second-order relations - that is, processing the specific distances among the features. Previous research has shown that adults use all of these three types of configural processing: They have a remarkable ability to detect faces among a sample of other visual stimuli on the basis of first-order relations (Moscovitch, Winocur, \& Behrmann, 1997). When adults detect the first-order relations of a face, they tend to process the face as a gestalt (holistic processing), which makes it harder to process individual features. This effect was demonstrated by the "composite face effect" (e.g., Young, Hellaway, \& Hay, 1987), in which the top half of a face can be recognized correctly when it is presented in isolation, but that recognition is significantly slower when the top half is combined with the bottom half of a different face. Holistic processing of faces has also been demonstrated by the "part-whole recognition effect" (Farah et al., 1998; Tanaka \& Farah, 1993), in which adults are more accurate in recognizing the identity of a feature when it is presented in the context of the whole face than when it is presented as an isolated feature. Evidence for holistic face processing also comes from studies where analytical and holistic processing were induced by a categorization task (Schwarzer, 2000). Here, a method from research on the processing of nonfacial visual stimuli was adopted for the domain of 
face processing (Ward, 1989; Ward \& Scott, 1987). Participants were asked to assign faces to two categories. In this task, they were free to categorize the faces analytically, by focusing on one specific feature, or holistically, by taking the overall similarity relations of the faces into account. The definition of holistic processing in terms of overall similarity relations is explained by Kemler Nelson (1989) as follows: Overall similarity relations are holistic insofar as

the stimulus representations that are accessed in processing are accessed as wholes, rather than as a concatenation of independent properties. ... When stimuli are compared, they are compared as wholes and the relation that is internally computed is their overall similarity. These holistic representations are not decomposed while doing computations and arriving at a response during the processing episode (p. 374).

Thus, the definition of holistic processing in terms of overall similarity relations also stresses the basic idea of gluing together the facial features into a gestalt although, in principle, overall similarity relations can be computed using independent processing with additive integration. Schwarzer's results showed that about $60 \%$ of adults categorized the faces holistically on the basis of overall similarity relations. The other participants based their categorization on single facial features and therefore processed the faces analytically. In children 6-11 years of age, however, the analytical face processing mode predominated (Schwarzer, 2000, 2002). Since all faces share the same first-order relations, recognition of individual faces requires the processing of more subtle variations such as the encoding of second-order relations. As shown by Haig (1984), adults can detect variations in the spatial distances among internal features as small as a visual angle of $1 \mathrm{~min}$.

The fact that analytical processing (e.g., the processing of a single facial feature independently of the facial context, also called componential or piecemeal processing) is also involved in face recognition is well documented. Tanaka and Farah (1993) have shown that individual features can be recognized with moderate accuracy, even when presented in isolation or in the context of a scrambled face. Tanaka and Sengco (1997) also acknowledged the influence of individual facial parts for face processing. Nevertheless, the authors emphasized the interaction between analytical and configural processing of the face because their results also showed that the alteration of facial configurations interfered with the retrieval of facial features, which was not the case with inverted faces or nonface stimuli. Wenger and Ingvalson (2002) also emphasized the analytical character of face processing. They demonstrated that holistic responses do not reflect processing at the first stage of information processing, as has been proposed by Farah et al. (1998) but instead reflect a bias at the decisional stage.

Thus, previous research has shown that both the configural and the analytical processing route exist in face processing. However, it is still not known what specific information, used at the stage of visual encoding, is associated with the different modes of face processing. The most direct method to study the stage of visual encoding is to analyze how people examine a face visually - that is, to analyze their gaze behavior during face processing. The following section describes what is known about people's gaze behavior when they process faces.

\section{Research on Gaze Behavior During Face Processing}

Previous studies on gaze behavior during face processing consist on the one hand of clinical studies (e.g., Cutting, 1990; Manor et al., 1999; Mertens, Siegmund, \& Grüsser, 1993; Mogg, Millar, \& Bradley, 2000; Shimizu et al., 2000) and on the other hand of studies that investigated gaze behavior and face processing in healthy populations. We will focus on the latter. Research on gaze behavior during face processing in healthy populations is in high agreement that participants fixate first and foremost the eyes and mouth when no instructions are given prior to viewing the faces (Groner, Walder, \& Groner 1984; Janik, Wellens, Goldberg, \& Dell'Osso, 1978; Yarbus, 1967). Jeffreys, Tukmachi, and Rockley (1992) studied the relationship between evoked potential and fixation position with facial images and concluded that the point between both eyes might be the position from which optimal processing occurs.

The study by Henderson, Falk, Minut, Dyer, and Mahadevan (2001) provides evidence that the fixation of central facial features, such as the eyes, nose, and mouth takes place not only when recognizing but also when encoding faces. The authors also compared fixation behavior when participants were asked to encode and recognize upright and inverted faces. Although the authors observed the inversion effect (e.g., Yin, 1969) — faces in the upright condition were recognized more easily and correctly than inverted ones - there was no effect with regard to fixation behavior. Fixation behavior for upright and inverted faces was very similar. This is the first study where a connection could be established between gaze behavior and configural, as well as analytical, processing. According to Tanaka and Farah (1993) and Farah et al. (1998), the inversion effect is accounted for by the holistic processing of upright faces and the analytical processing of inverted faces. Since, according to Henderson et al., the fixations for upright and inverted presentations of faces were similar, one could be led to assume that the different processing strategies do not correspond to a specific gaze behavior. Studies concerning gaze behavior during face processing in children exist, to our knowledge, only for clinical groups (e.g., van der Geest, Kemner, Verbaten, \& van Engeland, 2002). Whereas several studies with healthy children examined analytical and configural face processing in the course of development (e.g., Carey, 1996; Schwarzer, 2000, 2002; Tanaka, Kay, Grinnell, Stansfield \& Szechter, 1998), none of them studied gaze behavior, as well. Also, there are virtually no studies on children's gaze behavior when 
simply looking at faces. Thus, there has not yet been any systematic study of gaze behavior during analytical and configural face processing for any age groups. The aim of the present study was therefore to shed light on the stage of visual encoding when faces are processed analytically or, as one type of configural processing, holistically, and to analyze the corresponding gaze behaviors.

To address this question, we used the face-categorization task (Schwarzer, 2000; Ward \& Scott, 1987). Since previous studies had shown that the categorization task induces the analytical processing mode in most children 6-10 years of age (Schwarzer, 2000), we also studied gaze behavior during analytical and holistic processing in two groups of children. By studying children and adults, we aimed to induce a comparable frequency of analytical and holistic processors so that gaze behavior could be analyzed in these groups. Our hypotheses concerning the correspondence between mode of face processing and gaze behavior were derived from the concept of analytical and holistic processing. We assumed in the case of analytical processing - that is, focusing upon one facial feature - that processing would correspond to more and longer fixations of the specific area of the particular feature than to other areas of the face. Defining holistic processing as comparing faces according to their overall similarity - that is, their entire gestalts - we predicted that holistic processors' gaze behavior would be less feature specific and more dispersed over different or larger facial areas to obtain as much information about the whole face as possible.

\section{EXPERIMENT 1}

\section{Method}

We adopted a method developed by Ward and Scott (1987) to differentiate between analytical and holistic modes of face processing (see also Schwarzer, 2000). Children and adults were instructed to assign faces to two categories. The categories were constructed so as to allow either an analytical (focusing on a single feature) or holistic categorization of the faces. Gaze behavior was recorded during the entire categorization task.

Participants. Three age groups, 24 6- to 8 -year-olds (median $=$ 7.5 years, range $=6.0-8.10 ; 11$ female, 13 male), 209 - to 10 -yearolds $($ median $=10.6$ years, range $=9.9-10.11 ; 13$ female, 7 male $)$, and 20 adults (median $=24$ years, range $=17-35 ; 10$ female, 10 male) participated in this experiment. The children were from middle- to upper-middle-class families and attended elementary schools in Tübingen, and the adults were mostly undergraduates at the University of Tübingen.

Apparatus. The SMI remote eyetracker (refresh rate $50 \mathrm{~Hz}$ ) was used to track eye movements (spatial accuracy of gaze direction $0.5^{\circ}-1^{\circ}$ ). This operates by illuminating the eye with infrared light and monitoring its reflection off components of the eye via a video camera. The stimuli were displayed via a PC (single Pentium II processor, 512 MB RAM) on a 21-in. monitor (with a resolution of $1024 \times 768$ pixels) that was mounted together with the eyetracking camera and the infrared light source inside a so-called gaze-catch box. Gaze behavior was recorded on a second computer that was connected to the eyetracking computer. The two computers communicated via serial connection to provide synchronization of stimulus onset/offset and start and stop of recording of the gaze behavior. The gaze-catch box had a $16 \times 2$-cm slit where the participants could look inside. A chinrest was mounted below the slit so that the participants' head movements were reduced to a minimum.

Stimuli. The stimuli consisted of schematically drawn faces, presented on the computer monitor at a visual angle of $7.4^{\circ} \times 8^{\circ}$ on a light gray background. This visual angle enabled information of the entire face to be processed from any point of the internal section of the face. The stimulus faces were derived from two prototypical faces, a child and an adult face, which are presented in Figure 1. The average estimated age was 5 and 26 years for the child and adult faces, respectively.

The faces varied according to four features. The typical child face had large round eyes, a round nose, a small mouth, and a round outline. The adult face was characterized by more oval eyes, a narrow nose, a wide mouth, and an oval outline (see Table 1). A middle level between the two extremes was also constructed for each feature. Two categories of four child and four adult faces were formed on the basis of these feature variations (see Table 1). Each face shared a different cluster of three features with one of the prototypical faces, and the remaining feature was the middle level between the extreme levels of the prototypes. The family resemblance category structure allowed the participants to learn category membership by processing the faces either holistically or analytically. If the faces were compared on the basis of the clusters of the three characteristic features (overall similarity), these features were responsible for the way the face was categorized. This processing mode was our operationalization of holistic processing. If, by contrast, a single feature was processed (analytical processing), any one of the four features might have been used for the purpose of categorization. Focusing exclusively on a single facial feature leads to a $75 \%$ rate of correct categorization (e.g., a participant who focused on the eyes would sort Faces 1-3 into Category A and Faces 5-7 into Category B, and Faces 4 and 8 would be treated as exceptions). Similar predictions could be made for an analytical focus on one of the other three features (nose, mouth, or outline). Eight test faces were constructed to determine whether the categorization was based on a holistic or an analytical learning process (see Table 2). The structure of the features presented in Table 2 makes it possible to differentiate between holistic and analytical processing from each participant's categorizations of the test faces. For example, if only the eyes were considered for purposes of categorization, Face 1 would be assigned to Category B and Face 2 to Category A. In contrast, the opposite categorization would be due to a holistic procedure. The same reasoning applies to each of the four features.

To show that the salience of the different facial features is comparable, no single facial feature should overpower all the other features. Therefore, a dissimilarity judgment study was carried out that showed that, regardless of which features are considered, the test faces (see Table 2) will always be judged as being more similar to the category prototype they match on three features than to the contrasting prototype they match on the remaining feature. The study is described in detail in Schwarzer (2000).

Procedure. The participants were tested individually. Prior to the experiment, they were told that their gaze behavior would be recorded, and the eyetracker was calibrated once by a 9-point calibration technique. The experiment was divided into training and test phases. In the training phase, the eight faces from Categories A and $\mathrm{B}$ (see Table 1) were presented in four random sequences (a total of 32 training trials for each individual). Before the presentation of each face, a small white fixation cross appeared at the center of the monitor. Following the presentation of each face, the participant was asked to indicate whether the face belonged to Category A or B. The participants indicated category membership by using two small cardboard cards in their right and left hands that could be differentiated haptically. This method was chosen in order to ensure that gaze recording was not affected by possible head movements accompanying speech. One of the cards (representing, e.g., Cate- 

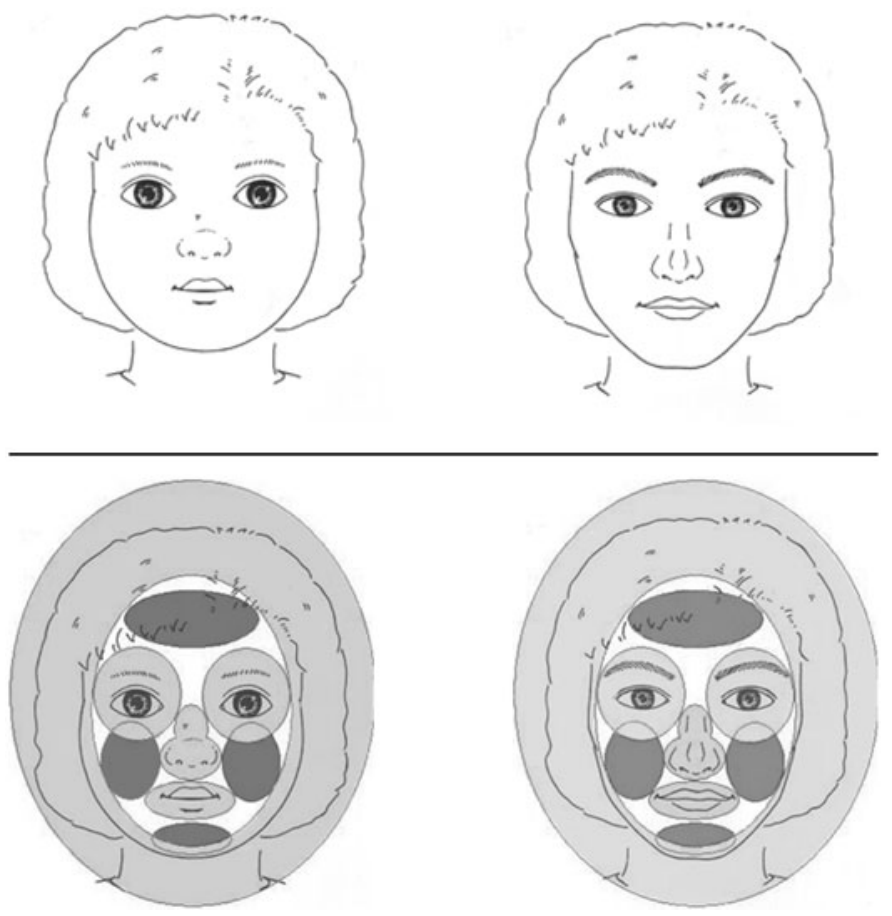

Figure 1. Experiment 1: Child and adult face (upper panel); facial regions defined for the analyses of gaze behavior (lower panel).

gory A) was covered with sponge rubber and the other one (Category B) with sandpaper. Once the participant gave the answer, the experimenter pressed a keyboard button to stop eye-movement recording. The experimenter made sure to stop the eye-movement recording reliably and gave feedback about the correctness of the categorization after each trial. Upon pressing the button, the face disappeared, and the fixation cross reappeared on the screen until the next trial was started by the experimenter. Thus, gaze behavior was recorded from stimulus onset until the experimenter pressed a button following the participant's response. In the subsequent test phase, the training faces and the test faces were presented in two random sequences. Again, the participant's task was to categorize the faces. This time, however, no feedback was given. Gaze behavior was again recorded.

Table 1

Facial Categories With Family Resemblance Structure (Training Faces, Presented During Training and Test)

\begin{tabular}{|c|c|c|c|c|}
\hline Faces & Eyes & Nose & Mouth & Outline \\
\hline \multicolumn{5}{|c|}{ Category A } \\
\hline 1 & round & round & small & medium \\
\hline 2 & round & round & medium & round \\
\hline 3 & round & medium & small & round \\
\hline 4 & medium & round & small & round \\
\hline Prototype A & round & round & small & round \\
\hline \multicolumn{5}{|c|}{ Category B } \\
\hline 5 & oval & narrow & wide & medium \\
\hline 6 & oval & narrow & medium & oval \\
\hline 7 & oval & medium & wide & oval \\
\hline 8 & medium & narrow & wide & oval \\
\hline Prototype B & oval & narrow & wide & oval \\
\hline
\end{tabular}

\section{Results and Discussion}

Face processing data. As in Schwarzer (2000) and Ward and Scott (1987), a learning criterion of 12 out of 16 correct categorizations of the training faces presented during the test phase was used to classify a participant as a successfully trained learner. This criterion served two purposes. First, it required more correct responses than could be expected by chance (binomial $p<.05$ ). Second, analytical learners who might only have acquired category information about the characteristic value of a particular feature but not about the exceptions to the rule could still meet the learning criterion. With the exception of four 6- to 8-year-olds, all participants reached the learning criterion. In the following analyses, only those participants who reached the learning criterion were considered.

The analyses for the type of categorization referred exclusively to the categorizations of the $2 \times 8$ test faces presented during the test phase. The general criterion for the diagnosis of an analytical categorization strategy was that at least 14 of the 16 test faces had to be processed by focusing on the same facial feature. Analogously, for the diagnosis of a holistic categorization strategy, at least 14 of the 16 test faces had to be classified on the basis of overall similarity.

The distribution of analytical and holistic processing strategies in the different age groups is depicted in Table 3. As can be seen in Table 3, in children and even in adults, the number of analytical processors was substantially 
Table 2

Structure of Test Faces, Presented During Test Only

\begin{tabular}{cllll}
\hline Test Face & Eyes & Nose & Mouth & Outline \\
\hline 1 & oval & round & small & round \\
2 & round & narrow & wide & oval \\
3 & round & narrow & small & round \\
4 & oval & round & wide & oval \\
5 & round & round & wide & round \\
6 & oval & narrow & small & oval \\
7 & round & round & small & oval \\
8 & oval & narrow & wide & round \\
\hline
\end{tabular}

higher than the number of holistic processors. The participants of the "other" group also focused on single facial features but did not use the same feature consistently. In contrast to our expectations, assignment to the different processing strategies was independent of age $\left[\chi^{2}(4)=1.17, p>.05\right]$. Nevertheless, holistic processors could be determined in each age group, and gaze behavior could be analyzed between the different groups of processors. In the following analyses of gaze behavior and face-processing strategies, the participants of the "other" group were excluded because only homogenous groups of analytical and holistic processors were to be compared.

Gaze behavior data. The analyses of the gaze behavior referred exclusively to the $2 \times 8$ training faces and $2 \times 8$ test faces (a total of 32 faces) presented during the test phase. For each trial, we obtained the fixations from the raw eye-movement data by using a dispersion algorithm (Stark \& Ellis, 1981) with a minimum fixation time of $100 \mathrm{msec}$ and a dispersion area of $.5^{\circ}$. Fixations were computed from the onset of face presentation until the response was given.

We compared the fixation behavior of analytical and holistic processors in the regions where the fixations occurred with respect to gaze time and number of fixations. We also analyzed total gaze time and reaction time per group of processors independently of facial region, as well as the size of the entire fixation area used. These analyses are described in detail below.

Distribution of gaze time over facial regions. To examine the location of the fixations, we defined seven ellipse-shaped regions, consisting of the eyes, the nose, the mouth, the facial outline, the forehead, the cheeks and the chin (see Figure 1, lower panel).

The sizes of the different regions, expressed as degree of visual angle, are depicted in Table 4 . For all of the 32

Table 3

Experiment 1: Number of Analytical and Holistic Processors in the Different Age Groups

\begin{tabular}{|c|c|c|c|c|c|c|}
\hline \multirow[b]{2}{*}{ Age Group } & \multicolumn{4}{|c|}{ Analytical } & \multirow[b]{2}{*}{ Holistic } & \multirow[b]{2}{*}{ Other } \\
\hline & Eyes & Nose & Mouth & Outline & & \\
\hline $6-8$ years & 3 & 3 & 2 & 4 & 3 & 5 \\
\hline $9-10$ years & 3 & 5 & 1 & 2 & 4 & 5 \\
\hline Adults & 2 & 2 & 0 & 8 & 5 & 3 \\
\hline
\end{tabular}

faces, we computed the gaze time spent on the different facial regions. Gaze time was calculated by cumulating the fixation times over all 32 trials of the test phase and dividing by the total number of 32 faces. From this, it follows that, even though fixation time was defined by a minimum of $100 \mathrm{msec}$ and a dispersion area of $.5^{\circ}$, gaze time could be less than $100 \mathrm{msec}$.

Scoring remained constant over all $2 \times 16$ faces for the central feature regions such as the eyes, nose, mouth, and facial outline. The remaining regions of the forehead, cheeks, and chin showed overlaps with the central regions. This varied to a very low degree among the 16 different faces ( $1 / 4^{\circ}$ on average). Therefore, here, we only computed the gaze time for the parts of the regions that did not overlap. Table 5 shows how gaze time was distributed over the different facial regions. As can be seen, participants' gaze time is mostly divided between the facial regions of the eyes, nose, and mouth, followed by the cheeks, chin, outline, and finally the forehead. An analysis of variance (ANOVA) of gaze time for the within-subjects factor facial region (eyes, nose, mouth, outline, forehead, cheeks, chin) and the between-subjects factors processing strategy (analytical, holistic) and age (three age groups) revealed a significant result for facial region $[F(6,198)=19.86, p<$ $.01]$ and a significant interaction between facial region and processing strategy $[F(24,198)=3.06, p<.01]$ but no significant interaction between facial region and age group and no significant threefold interaction $[F(12,198)<1$, $F(42,198)=1.27, p>.05]$. Concerning the betweensubjects factors, there was no significant effect of processing strategy $[F(4,33)<1]$ and no effect of age $[F(2,33)=$ $2.37, p>.05]$.

As can be seen in Table 5, holistic processors directed their gaze longest to the facial regions of the eyes and nose (336 and $258 \mathrm{msec}$, respectively), whereas they fixated the other regions within a range of 8-79 msec only. Analyticaleye processors looked longest at the eyes (578 vs. $74 \mathrm{msec}$ on average at the other regions), analytical-nose processors looked longest at the nose (480 vs. $57 \mathrm{msec}$ on average at the other regions), and the analytical-mouth processors looked longest toward the mouth region $(516 \mathrm{msec}$ vs. $84 \mathrm{msec}$ on average at the other regions), followed by the adjacent facial region of the chin $(218 \mathrm{msec})$. The analytical-outline processors fixated the nose and the eyes longest (363 and $215 \mathrm{msec}$, respectively, vs. $55 \mathrm{msec}$ on average at the other regions). Thus, by comparing the gaze time of the holistic processors with that of the analytical processors, a clear differentiation between holistic processors and analytical-eye, analytical-nose, and analytical-mouth processors could be achieved. While the holistic processors looked longest at the eyes and nose, the analytical processors fixated those facial features longest that they subsequently processed - that is, the eyes, nose, and mouth. The gaze time of the remaining analytical-outline processors, however, showed overlaps with the holistic processors' gaze times. Here, nose and eyes were fixated longest. 
Table 4

Experiments 1 and 2: Degree of Visual Angle of the Facial Regions Defined in the Stimulus Faces

\begin{tabular}{|c|c|c|c|c|c|c|c|}
\hline & Eyes (Each) & Nose & Mouth & Outline & Forehead & Cheeks (Each) & Chin \\
\hline Experiment 1 & $1.7^{\circ} \times 1.7^{\circ}$ & $1.2^{\circ} \times 1.3^{\circ}$ & $1.8 \times .7^{\circ}$ & $\begin{array}{c}7.4^{\circ} \times 8.0^{\circ} \\
(\text { external) } \\
4.2^{\circ} \times 5.0^{\circ} \\
\text { (internal) }\end{array}$ & $3.0^{\circ} \times 1.0^{\circ}$ & $1.4^{\circ} \times 1.4^{\circ}$ & $1.6^{\circ} \times .6^{\circ}$ \\
\hline Experiment 2 & $1.9^{\circ} \times 1.4^{\mathrm{o}}$ & $1.7^{\circ} \times 1.8^{\circ}$ & $2.4^{\mathrm{o}} \times .7^{\mathrm{o}}$ & $\begin{array}{c}7.5^{\circ} \times 7.3^{\circ} \\
(\text { external }) \\
4.9^{\circ} \times 4.9^{\circ} \\
(\text { internal })\end{array}$ & $3.2^{\circ} \times 1.3^{\circ}$ & $1.2^{\circ} \times 1.4^{\circ}$ & $1.9^{\circ} \times .7^{\mathrm{o}}$ \\
\hline
\end{tabular}

\section{Distribution of the average number of fixations} over facial regions. Analogously to the gaze time, we calculated the average number of fixations by cumulating the number of fixations over all 32 trials of the test phase and dividing by the total number of 32 faces. An ANOVA of the average number of fixations for the within-subjects factor facial region (eyes, nose, mouth, outline, forehead, cheeks, chin) and the between-subjects factors processing strategy (analytical, holistic) and age (three age groups) revealed a significant result for facial region $[F(6,198)=$ 23.17, $p<.01$ ], a significant interaction between facial region and processing strategy $[F(24,198)=2.91, p<$ $.01]$, but no significant interaction between facial region and age group and no threefold interaction $[F(12,198)<1$; $F(42,132)=1.31, p>.05]$. There was no effect of processing strategy $[F(4,33)<1]$, but the between-subjects factor age group reached the level of significance $[F(2,33)=3.74, p<.05]$. Overall, the children (4.74 and 4.83 fixations for the two age groups) showed more fixations than did the adults (3.64 fixations). With regard to the interaction between facial region and processing strategy, Table 5 shows that the distribution of the average number of fixations over the seven facial regions was very similar to the distribution of gaze time over the different regions. Again, holistic processors fixated the regions of the eyes and nose most often. The analytical-eye processors mainly fixated the eye region, the analyticalnose processors mainly fixated the nose region, and the analytical-mouth processors mainly fixated the mouth region. With regard to the analytical-outline processors, the nose and eye regions were fixated most, as can be seen above in the analyses of gaze time.
Overall gaze time and reaction time. An ANOVA of gaze time, independent of the different facial regions used, was carried out with the between-subjects factors of processing strategy (analytical, holistic) and age (three age groups). This revealed a significant effect of age $[F(2,41)=3.79, p<.05]$, but no effect of processing strategy $[F(1,41)=1.07, p>.05]$, and no significant interaction $[F(2,41)<1]$. The effect of age showed that gaze time decreased significantly with increasing age $(1,240 \mathrm{msec}$ for the 6 - to 8 -year-olds, $1,175 \mathrm{msec}$ for the 9- to 10-year-olds, and $877 \mathrm{msec}$ for the adults). To understand why overall gaze time differed with age but gaze time in the different facial regions did not (see above), we subtracted the gaze time in all facial regions from the overall gaze time. We called this measure rest and computed an ANOVA of rest $\times$ age group as the between-subjects factor. Although the "age group" factor did not reach the level of significance, there was a tendency for the children to look longer at the facial areas that lay between the defined regions (rest) $[F(2,46)=$ $2.52, p=.09]$. The average gaze time rest was $270 \mathrm{msec}$ for the 6- to 8-year-olds, $238 \mathrm{msec}$ for the 9- to 10 -yearolds, and $160 \mathrm{msec}$ for the adults.

The analyses of the reaction times showed comparable results: Here, age and the interaction between age and processing reached significance $[F(2,41)=10.76, p<$ $.01, F(2,41)=3.71, p<.05]$, but processing strategy $\operatorname{did} \operatorname{not}[F(1,41)<1]$. Analogously to the gaze times, the reaction times also decreased with age $(2,182 \mathrm{msec}$ for the 6- to 8-year-olds, $1,951 \mathrm{msec}$ for the 9- to 10 -yearolds, 1,424 msec for the adults). These age differences were slightly more marked for the holistic processors

Table 5

Experiment 1: Gaze Time (Average of the Cumulated Fixation Times of the 32 Test Faces in Milliseconds) and Average Number of Fixations (in Parentheses) Spent on the Different Facial Areas by Analytical and Holistic Processors

\begin{tabular}{|c|c|c|c|c|c|c|c|}
\hline & Eyes & Nose & Mouth & Outline & Forehead & Cheeks & Chin \\
\hline \multicolumn{8}{|l|}{ Analytical } \\
\hline Eye & $578(2.82)$ & $148 \quad(.78)$ & $37(.18)$ & $65(.27)$ & $42(.25)$ & $145(.61)$ & $8 \quad(.04)$ \\
\hline Nose & $200 \quad(.87)$ & $480(1.91)$ & $75(.38)$ & $20(.46)$ & $3(.02)$ & $38(.16)$ & $8 \quad(.05)$ \\
\hline Mouth & $89 \quad(.54)$ & $144 \quad(.83)$ & $516(2.3)$ & $20(.03)$ & $1(.01)$ & $33(.20)$ & $218 \quad(.93)$ \\
\hline Outline & $215(1.17)$ & $363(1.67)$ & $127(.61)$ & $26(.15)$ & $5(.02)$ & $99(.52)$ & $18(.09)$ \\
\hline Holistic & $336(1.90)$ & $258(1.54)$ & $55(.36)$ & $79(.14)$ & $11(.08)$ & $61(.35)$ & $8(.005)$ \\
\hline Total & 1,418 & 1,393 & 810 & 210 & 62 & 376 & 260 \\
\hline
\end{tabular}


$(2,006,2,455$, and $1,287 \mathrm{msec})$ than for the analytical processors $(2,226,1,768$, and $1,480 \mathrm{msec})$.

In sum, Experiment 1 showed that analytical and holistic face processing corresponds to specific gaze behavior in terms of different locations of fixations. Whereas holistic processors fixated most and longest the areas of the eyes and nose, analytical processors mainly focused their fixations on the particular facial feature they used for subsequent processing. However, the present experiment leaves open the question of whether the results are really representative for face processing since the analyses of the gaze behavior were based on an atypically high proportion of adults who used an analytical processing strategy. According to the previous literature, analytical processors are usually in the minority. Therefore, in the next experiment we attempted to facilitate the more typical holistic processing of faces. To this end, we used photographs of real faces as stimuli instead of schematically drawn faces because in photos the part-based structure-which usually induces analytical processing - is less pronounced.

\section{EXPERIMENT 2}

\section{Method}

We used the same categorization task described above, in Experiment 2 , to study the gaze behavior of children and adults when processing more-natural-looking faces analytically or holistically.

Participants. Three age groups, 27 6- to 7-year-olds $($ median $=$ 7.0 years, range $=6.1-7.11 ; 16$ female, 19 male), 279 - to 10 -yearolds $($ median $=10.0$ years, range $=9.0-11.3 ; 13$ female, 14 male $)$, and 21 adults (median $=24$ years, range $=21-39 ; 10$ female, 11 male) participated in this experiment.

Apparatus. The apparatus was the same as described in Experiment 1.

Stimuli. The stimuli consisted of photographs of real faces. As in Experiment 1, the faces were presented on a computer monitor at a visual angle of $7.5^{\circ} \times 7.3^{\circ}$ on a light-gray background. Analogously to the schematic faces, the stimulus faces were derived from two prototypical faces. The prototypical faces were also based on a child face and an adult face, 5 and 29 years of age, respectively, as depicted in Figure 2. Four features were varied in the prototypical faces: eyes, nose, mouth, and facial outline, similarly to the schematic faces. As in the schematic faces, there were three levels for each of the features (see Table 1). Two categories of four child faces and four adult faces were formed on the basis of these variations in features. Also, eight test faces were constructed according to the principle of the schematically drawn faces (see Table 2). The faces were constructed by using a morphing software developed by Vetter and Troje (1997). It was found that no single facial feature was so salient that it overpowered all of the other features. This study is described in detail in Schwarzer and Korell (1999).

Procedure. The procedure was the same as described in Experiment 1.

\section{Results}

Face processing data. Seventy-seven percent $(n=$ $21)$ of the 6 - to 7 -year-olds, $85 \%(n=23)$ of the 9 - to 10 year-olds, and $90 \%(n=19)$ of the adults reached the learning criterion used in Experiment 1. There was a significant relationship between age and reaching the learning criterion $\left[\chi^{2}(2)=8.55, p<.05\right]$, showing that the ability to differentiate the facial categories increased
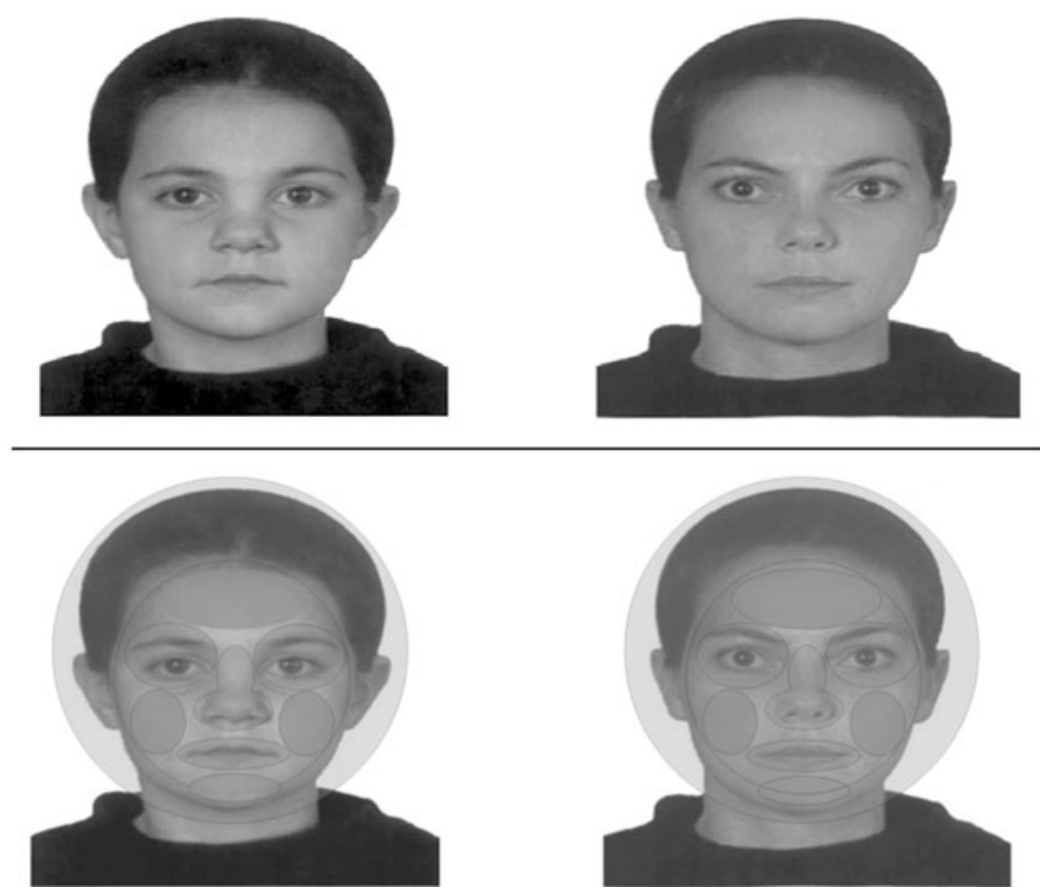

Figure 2. Experiment 2: Child and adult face (upper panel); facial regions defined for the analyses of gaze behavior (lower panel). 
with age. For all further analyses, only children and adults who met the learning criterion are considered.

We used the general condition to determine an analytical or holistic processing mode described in Experiment 1 and found that $24 \%$ of the 6 - to 7 -year-olds processed the faces analytically, 19\% used the holistic processing mode, and 57\% used the "other" strategy (see Table 6). Of the 9- to 10 -year-olds, $26 \%$ processed the faces analytically, $44 \%$ used the holistic processing mode, and $30 \%$ were classified as "other." Of the adults, $21 \%$ processed the faces analytically, $63 \%$ used the holistic processing mode, and $16 \%$ used the "other" strategy. A $\chi^{2}$ test indicated a nearly significant relationship between age and processing mode $\left[\chi^{2}(4)=8.96\right.$, $p=.06]$. Most of the youngest children used the analytical processing mode or the "other" strategy that also indicates a kind of analysis because the children focused on single features but did not use the same features consistently. Older children, and adults in particular, predominantly used the holistic processing mode that is typical for face processing. Thus, there was a trend toward the previously reported developmental shift from an analytical to a holistic mode of processing (Schwarzer, 2000). As in Experiment 1, the participants in the "other" group were excluded in the analyses of gaze behavior.

Gaze behavior data. By using the same method as in Experiment 1, we analyzed children's and adults' gaze behavior when processing the faces analytically or holistically.

Distribution of gaze time over facial regions. To examine which areas of the face had been fixated by analytical and holistic processors, as in Experiment 1, we defined seven ellipse-shaped regions consisting of the eyes, the nose, the mouth, the facial outline, the forehead, the cheeks, and the chin (see Figure 2, lower panel). The sizes of the different regions expressed as degree of visual angle are shown in Table 4. By using the same method described in Experiment 1, we calculated the gaze time spent on the different facial regions for all of the 32 faces and ran an ANOVA of gaze time for the within-subjects factor facial region (eyes, nose, mouth, outline, forehead, cheeks, chin) $\times$ between-subjects factors processing strategy and age. The analysis revealed a significant result for facial region $[F(6,168)=9.69, p<$ $.01]$ and more importantly, a significant interaction between region and strategy $[F(24,168)=2.93, p<.01]$. The interaction between region and age and the three-

Table 6

Experiment 2: Number of Analytical and Holistic Processors in the Different Age Groups

\begin{tabular}{|c|c|c|c|c|c|c|}
\hline \multirow[b]{2}{*}{ Age Group } & \multicolumn{4}{|c|}{ Analytical } & \multirow[b]{2}{*}{ Holistic } & \multirow[b]{2}{*}{ Other } \\
\hline & Eyes & Nose & Mouth & Outline & & \\
\hline $6-7$ years & 2 & 0 & 1 & 1 & 5 & 12 \\
\hline $9-10$ years & 2 & 1 & 2 & 1 & 10 & 7 \\
\hline Adults & 2 & 1 & 0 & 1 & 12 & 3 \\
\hline
\end{tabular}

fold interaction did not reach the level of significance $[F(12,168)=1.52, p>.05 ; F(36,168)=1.01, p>.05$. $]$ With regard to the between-subjects factors, age was not significant $[F(2,28)=1.78, p>.05]$, but processing strategy and the interaction between processing strategy and age did reach the level of significance $[F(4,28)=$ $2.74, p<.05 ; F(6,28)=2.81, p<.05]$. As depicted in Table 7 , the interaction between processing strategy and gaze time is relatively similar to the pattern observed in Experiment 1: The holistic processors looked longest at the nose $(303 \mathrm{msec})$, followed by the eyes $(165 \mathrm{msec})$, and the mouth $(158 \mathrm{msec})$ in comparison with the other regions, which they looked at for $54 \mathrm{msec}$ on average. Analytical-eye processors directed their gaze longest to the region of the eyes (534 vs. $126 \mathrm{msec}$ on average in comparison to the other regions). Analytical-nose processors looked longest at the nose (828 vs. $97 \mathrm{msec}$ on average spent looking at the other regions). The analytical-mouth processors directed their gaze longest toward the mouth and adjacent chin region ( $357 \mathrm{msec} ; 464$ vs. $80 \mathrm{msec}$ on average in comparison with the other regions). The analyticaloutline processors, fixated the nose longest (443 vs. $108 \mathrm{msec}$ on average spent looking at the other regions). All in all, as shown in Table 7, the holistic processors' gaze time was shorter than that of the analytical processors, whereby this effect was different in each of the three age groups (see Table 7, note).

Distribution of the average number of fixations over facial regions. Analogously to Experiment 1, we ran an ANOVA of the average number of fixations for the within-subjects factor facial region (eyes, nose, mouth, outline, forehead, cheeks, chin) $\times$ the between-subjects factors processing strategy and age. The analysis showed a significant result for facial region $[F(6,168)=10.63$, $p<.01]$, and a significant interaction between facial region and processing strategy $[F(24,168)=1.97, p<$ $.01]$. Again, the effects of the interaction between region and age, as well as the threefold interaction were not significant $[F(12,168)=1.62, p>.05 ; F(36,168)<1, p>$ $.05]$. With regard to the between-subjects factors, there was no significant effect of age $[F(2,28)<1, p>.05]$ or strategy $[F(4,28)=1.33, p>.05]$ and no interaction between age and strategy $[F(6,28)=1.61, p>.05]$. As shown in Table 7, holistic processors mostly used the region of the nose. The analytical-eyes processors used the region of the eyes most often, the analytical-nose processors and the analytical-outline processors mainly used the nose region, and the analytical-mouth processors mainly used the mouth region. Thus, as in Experiment 1, the analyses of gaze time and number of fixations allow a differentiation between holistic processors and analyticaleye, analytical-nose, and analytical-mouth processors: Whereas the holistic processors looked longest and mostly at the nose and eyes, the analytical processors fixated those facial features longest that they subsequently processed - that is, eyes, nose, and mouth. Again, the gaze behavior of the analytical-outline processors 
Table 7

Experiment 2: Gaze time (Average of the Cumulated Fixation Times of the 32 Test Faces in Milliseconds) and Average Number of Fixations (in Parentheses) Spent on the Different Facial Areas by Analytical and Holistic Processors

\begin{tabular}{|c|c|c|c|c|c|c|c|c|c|}
\hline & Eyes & Nose & Mouth & Outline & Forehead & Cheeks & Chin & & Total $^{*}$ \\
\hline \multicolumn{10}{|l|}{ Analytical } \\
\hline Eye & $534(2.36)$ & $361(1.70)$ & $148 \quad(.57)$ & $14(.10)$ & $19(.09)$ & $199(.63)$ & $(.05)$ & 1,287 & $(1,500,1,610,753)$ \\
\hline Nose & $130 \quad(.89)$ & $828(3.38)$ & $342(1.48)$ & 33 & $16(.06)$ & 16 & $43 \quad(.16)$ & 1,408 & $3,1,080)$ \\
\hline Mouth & $14 \quad(.08)$ & $61 \quad(.28)$ & $357(1.38)$ & $268(.93)$ & $\begin{array}{ll}0 & (0)\end{array}$ & $55(.28)$ & $464(1.16)$ & 1,219 & $, 1,508,0)$ \\
\hline Outline & $215(1.30)$ & $443(2.02)$ & $185 \quad(.88)$ & $25(.15)$ & $21(.16)$ & $110(.45)$ & $90 \quad(.34)$ & 1,089 & $88,1,346)$ \\
\hline Holistic & $165 \quad(.91)$ & $303(1.46)$ & $158 \quad(.74)$ & $48(.26)$ & $9(.06)$ & $120(.54)$ & $(.17)$ & 841 & $(1,134,828,731)$ \\
\hline Total & 1,058 & 1,996 & 1,189 & 389 & 66 & 499 & 648 & & \\
\hline
\end{tabular}

${ }^{*}$ Gaze times of the 6- to 7-year-olds, 9- to 10-year-olds, and adults, in that order.

was similar to that of the holistic processors. Both groups fixated the nose most and longest.

Overall gaze time and reaction time. An ANOVA of the overall gaze time, independently of the different facial regions used, was carried out with the between-subjects factors processing strategy and age. This showed a significant effect of age $[F(2,35)=7.92, p<.01]$ and strategy $[F(1,35)=9.64, p<.01]$, but no significant effect of the interaction between age and strategy $[F(2,35)=$ $1.62, p>.05]$. The effect of age revealed that gaze time decreased with increasing age $(1,926 \mathrm{msec}$ for the 6 - to 7 -year-olds, $1,469 \mathrm{msec}$ for the 9 - to 10 -year-olds, $1,418 \mathrm{msec}$ for the adults), and the effect of strategy showed that analytical processors' gaze time was significantly longer than that of the holistic processors $(1,824 \mathrm{msec}$ for analytical processors vs. $1,208 \mathrm{msec}$ for holistic processors). The analysis of the reaction times showed similar results: Age and strategy reached significance $[F(2,35)=6.99, p<.01 ; F(1,35)=6.37, p<$ $.01]$, whereas the interaction between these factors was not significant $[F(2,35)<1, p>.01]$. Again, reaction time decreased with increasing age $(2,758 \mathrm{msec}$ for the 6- to 7-year-olds, 2,210 msec for the 9- to 10-year-olds, $1,688 \mathrm{msec}$ for the adults), and analytical processing was associated with longer reaction times than was holistic processing $(2,556 \mathrm{msec}$ for analytical processors vs. $1,904 \mathrm{msec}$ for holistic processors).

\section{DISCUSSION}

The present study has shown that analytical and holistic face processing correspond to specific gaze behavior in terms of different locations of fixations. Whereas holistic processors fixated most and longest the areas of the eyes and nose, analytical processors mainly focused their fixations on the particular facial feature they used for subsequent processing. Thus, differences in analytical and holistic face processing already exist at the visual encoding stage and in the functional use of facial information.

In congruence with previous research on the information processing of faces, the present results showed that adults use both modes of face processing - analytical and holistic processing. When natural-looking faces were processed (as in Experiment 2), in accordance with the findings of other studies (e.g., Farah et al., 1998;
Schwarzer, 2000; Tanaka \& Farah, 1993; Young, Hellaway, \& Hay, 1987), the dominance of holistic processing over analytical processing could be observed. When, on the other hand, schematically drawn faces were processed (as in Experiment 1) most adults used the analytical strategy. One reason for this discrepancy may be that the schematic face stimuli encourage an analytical feature-matching strategy much more than do the natural face stimuli, because in the schematic stimuli the partbased structure is especially emphasized. The increase of holistic processors when processing natural-looking faces was also observable among the children. Whereas most children in Experiment 1 processed the faces analytically and only very few used a holistic processing mode, the children in Experiment 2 used the holistic processing mode more often, although still less than the adults.

The gaze behavior of all age groups in the present study was in line with previous studies on face processing; that is, all participants fixated only a few facial features. Moreover, some changes in gaze behavior were observable with increasing age. The average gaze times and the number of fixations used decreased with age and, consequently, the reaction times also decreased with age. Thus, in general, gaze behavior became more focused with increasing age both in terms of fixation duration and the number of fixations. Most important, in both experiments we found a significant interaction between the modes of processing and the areas of the face used for fixations, as well as a different frequency of analytical and holistic processors (especially in adults) in the two experiments. Holistic processors used the facial areas of the eyes and nose the most and longest. In contrast, analytical eye, nose, and mouth processors fixated the facial feature they used for further processing most and longest - that is, the eyes, nose, or mouth (or the adjacent chin region). However, for analytical processors who focused on the facial outline there was no direct link to gaze behavior. They did not fixate points of the facial outline primarily but instead mostly fixated the nose, similarly to participants with an analytical-nose processing strategy. In contrast to local features, such as the eyes, nose, and mouth, the outline of a face is a feature with global character, since it is the attribute of the face that shows its entirety. Apparently, the nose area used by 
the analytical-outline processors as well as the eyes and nose areas (i.e., the center of the face) used by the holistic processors are ideal starting points for gathering information about the face as a whole. It should be noted, however, that an interpretation of the correspondence between gaze behavior and mode of face processing does not show how the processed face is represented exactly. For example, a face can be represented configurally in terms of first- or second-order relational properties, and both kinds of representations can be associated with the same gaze behavior-that is, looking at the eyes and nose the most and longest.

Our findings that analytical and holistic face processing were accompanied by specific gaze behavior are not in line with Henderson et al.'s (2001) results on gaze behavior in the recognition of upright and inverted faces. It was shown by Henderson et al., that the facial features selected for fixation during recognition of an upright face, assumed to be supported by holistic processing, were very similar to those selected for fixation during recognition of an inverted face, assumed to be supported by analytical processing. One reason for the discrepancy in the results could lie in the use of different methods to study the relationship between analytical and holistic face processing and gaze behavior. Henderson et al. examined this relationship by studying face recognition performance and gaze behavior when faces were presented upright and inverted. Whereas previous research justifies using this inversion method to deduce analytical and holistic processing, it is not clear whether gaze behavior in the inverted condition can be ascribed only to analytical processing or whether other components are also relevant when looking at inverted faces. For example, it is possible that participants explicitly tried to scan the inverted faces in the same way as they scanned the upright faces. But our analyses of the participants' gaze referred exclusively to upright faces, and the different processing modes were induced directly via a categorization task.

Nevertheless, further experimentation is necessary to clarify to what extent the correspondence between gaze behavior and mode of face processing shown in the present study depends on the conceptualization of holistic processing in terms of overall similarity relations. Other studies focused on the conceptualization of holistic processing in terms of representing faces as unanalyzed psychological entities or in terms of processing featural and configural information simultaneously (see Wenger \& Ingvalsson, 2002, for an overview). Whether these conceptualizations are associated with gaze behavior that differs from the gaze behavior found in the present study should be investigated in the future. In Experiment 2, we found another important link between mode of face processing and gaze behavior. Here, holistic processors showed a significantly shorter overall gaze time and reaction time than did analytical processors. From this, it can be concluded that the holistic processing mode is reflected in gaze behavior as a fast and highly optimal gaze strategy (fixation of the center of the faces) in comparison with gaze behavior, which is associated with analytical processing. Analytical processing is accompanied by a longer gaze time and reaction time and very specific locations of fixations. This conclusion, however, is only supported by the results of Experiment 2. In Experiment 1, no differences in gaze behavior and reaction time were found between analytical and holistic processors. One reason for this result may be that in Experiment 1, the distributions of analytical and of holistic processors were so unequal that potential differences could not be observed.

In sum, the results of both experiments are in line with our expectations. They were derived from the theoretical concepts of analytical and holistic processing, under the assumption that analytical processing is accompanied by a more feature-specific gaze behavior than is holistic face processing. Thus, different modes of face processing such as analytical and holistic processing are reflected in differences in gaze behavior - that is, early on at the visual encoding stage.

\section{REFERENCES}

Bruyer, R., \& Coget, M. C. (1987). Features of laterally displayed faces: Saliency or top-down processing? Acta Psychologica, 66, 103114.

Carey, S. (1996). Perceptual categorization and expertise. In R. Gelman \& T. Kit-Fong Au (Eds.), Perceptual and cognitive development (pp. 49-69). San Diego: Academic Press.

Collishaw, S. M., \& Hole, G. J. (2000). Featural and configural processes in the recognition of faces of different familiarity. Perception, 29, 893-909.

CUTTING, J. (1990). The right cerebral hemisphere and psychiatric disorders. Oxford: Oxford University Press.

DiAmOND, R., \& CAREY, S. (1986). Why faces are and are not special: An effect of expertise. Journal of Experimental Psychology: General, 115, 107-117.

FARAH, M. J., TANAKA, J. W., \& Drain, H. M. (1995). What causes the face inversion effect? Journal of Experimental Psychology: Human Perception \& Performance, 21, 628-634.

FARAH, M. J., Wilson, K. D., DRAIN, [H.] M., \& TANaKa, J. N. (1998). What is special about face perception? Psychological Review, 105, 482-498.

FreIRE, A., LeE, K., \& Symons, L. A. (2000). The face inversion effect as a deficit in the encoding of configural information: Direct evidence. Perception, 29, 159-170.

Groner, R., Walder, F., \& Groner, M. (1984). Looking at faces: Local and global aspects of scan paths. In A. G. Gale \& F. Johnson (Eds.), Theoretical and applied aspects of gaze behavior research (pp. 523-533). Amsterdam: Elsevier.

HaIG, N. D. (1984). The effect of feature displacement on face recognition. Perception, 13, 505-512.

Henderson, J. M., FalK, R., Minut, S., Dyer, F. C., \& MahadeVAN, S. (2001). Gaze control for face learning and recognition in humans and machines. In T. Shipley \& P. Kellman (Eds.), From fragments to objects: Segmentation processes in vision (pp. 463-481). New York: Elsevier.

Janik, S. W., Wellens, A. R., Goldberg, M. L., \& Dell'Osso, L. F. (1978). Eyes as the center of focus in the visual examination of human faces. Perceptual \& Motor Skills, 47, 857-858.

Jefrreys, D. A., TuKMaChI, E. S. A., \& RockLEy, G. (1992). Evoked potential evidence for human brain mechanisms that respond to single, fixated faces. Experimental Brain Research, 91, 351-362.

Kemler Nelson, D. G. (1989). The nature and occurrence of holistic 
processing. In B. E. Shepp \& S. Ballesteros (Eds.), Object perception: Structure and process (pp. 357-386). Hillsdale, NJ: Erlbaum.

LEDER, H., \& BRUCE, V. (2000). When inverted faces are recognized: The role of configural information in face recognition. Quarterly Journal of Experimental Psychology, 53A, 513-536.

Macho, S., \& Leder, H. (1998). Your eyes only? A test of interactive influence on the processing of facial features. Journal of Experimental Psychology: Human Perception \& Performance, 24, 1486-1500.

Manor, B. R., Gordon, E., Williams, L. M., Rennie, C. J., Bahramali, H., Latimer, C. R., Barry, R. J., \& Meares, R. A. (1999). Gaze behavior reflects impaired face processing in patients with schizophrenia. Biological Psychiatry, 46, 963-969.

Maurer, D., Le Grand, R., \& Mondloch, C. J. (2002). The many faces of configural processing. Trends in Cognitive Sciences, 6, 255-260.

Mertens, I., Siegmund, H., \& Grüsser, O.-J. (1993). Gaze motor asymmetries in the perception of faces during a memory task. Neuropsychologia, 31, 989-998.

Mogg, K., Millar, N., \& Bradley, B. P. (2000). Biases in gaze behavior to threatening facial expressions in generalized anxiety disorder and depressive disorder. Journal of Abnormal Psychology, 109. 695-704.

Moscovitch, M., Winocur, G., \& Behrmann, M. (1997). What is special about face perception? Nineteen experiments on a person with visual object agnosia and dyslexia but normal face recognition. Journal of Cognitive Neuroscience, 9, 555-604.

SchWANinger, A., Lobmaier, J., \& Collishaw, S. M. (2002). Role of featural and configural information in familiar and unfamiliar face recognition. Lecture Notes in Computer Science, 2525, 643-650.

Schwarzer G. (2000). Development of face processing: The effect of face inversion. Child Development, 71, 391-401.

SchwARZER, G. (2002). Processing of facial and nonfacial visual stimuli in 2-5-year-old children. Infant \& Child Development, 11, 253-269.

Schwarzer, G., \& Korell, M. (1999). Development of face processing: Differences between schematic and photorealistic faces. Poster presented at the biennial meetings of the Society for Research in Child Development. Albuquerque, NM.

Schwarzer, G., \& Massaro, D. W. (2001). Modeling face identification processing in children and adults. Journal of Experimental Child Psychology, 79, 139-161.

SEARCY, J. H., \& BARTLETT, J. C. (1996). Inversion and processing of component and spatial-relational information in faces. Journal of Experimental Psychology: Human Perception \& Performance, 22, 904-915.

Shimizu, T., Shimizu, A., Yamashita, E., Iwase, M., Kajimoto, O., \&
KAWASAKI, T. (2000). Comparison of eye-movement patterns in schizophrenic and normal adults during the examination of facial affect displays. Perceptual \& Motor Skills, 91, 1045-1056.

STARK, L., \& ElLIS, S. R. (1981). Scanpath revisited: Cognitive models of direct active looking. In D. F. Fisher, R. A. Monty, \& J. W. Senders (Eds.), Gaze behavior: Cognition and visual perception (pp. 193226). Hillsdale, NJ: Erlbaum.

TANAKA, J. W., \& FARAH, M. J. (1993). Parts and wholes in face recognition. Quarterly Journal of Experimental Psychology, 46A, 225245.

Tanaka, J. W., Kay, J. B., Grinnell, E., Stansfield, B., \& Szechter, L. (1998). Face recognition in young children: When the whole is greater than the sum of its parts. Visual Cognition, 5, 479-496.

TAnaKa, J. W., \& SengCo, J. A. (1997). Features and their configuration in face recognition. Memory \& Cognition, 25, 583-592.

van der Geest, J. N., Kemner, C., Verbaten, M. N., \& van EngeLAND, H. (2002). Gaze behavior of children with pervasive developmental disorder toward human faces: A fixation time study. Journal of Child Psychology \& Psychiatry \& Allied Disciplines, 43, $669-678$.

VETTER, T., \& TroJe, N. (1997). Separation of texture and shape in images of faces for image encoding and synthesis. Journal of the Optical Society of America, 14, 2152-2161.

VIVIANI, P. (1990). Gaze behavior in visual search. Cognitive, perceptual and motor control aspects. In E. Kowler (Ed.), Eye movements and their role in visual and cognitive processes (pp. 353-393). Amsterdam: Elsevier.

WARD, T. B. (1989). Analytic and holistic modes of processing in category learning. In B. E. Shepp \& S. Ballesteros (Eds.), Object perception: Structure and process. Hillsdale, NJ: Erlbaum.

WARD, T. B., \& ScotT, J. G. (1987). Analytical and holistic modes of learning family-resemblance concepts. Memory \& Cognition, 15, 54.

Wenger, M. J., \& IngValson, E. M. (2002). A decisional component of holistic encoding. Journal of Experimental Psychology: Learning, Memory, \& Cognition, 28, 872-892.

YARBUS, A. L. (1967). Gaze behavior and vision. New York: Plenum.

YIN, R. K. (1969). Looking at upside-down faces. Journal of Experimental Psychology. 81, 141-145.

Young, A. W., Hellaway, D., \& Hay, D. (1987). Configural information in face perception. Perception, 16, 747-759.

(Manuscript received August 8, 2003; revision accepted for publication June 16, 2004.) 